\title{
Research on Flower Retrieval Based on Deep Learning
}

\author{
Yuan Niu ${ }^{a}$, Xuelong Hu ${ }^{\text {a, }}$, Shuhan Chen ${ }^{\mathrm{a}}$, Chenhui Yang ${ }^{\mathrm{b}}$ \\ a School of Information Engineering, Yangzhou University, Yangzhou 225127, China \\ b Jiangsu Haorun Electronic Technology Co., Ltd., Changzhou 213000, China \\ *Corresponding Author: xlhu@yzu.edu.cn
}

\begin{abstract}
Traditional flower retrieval system uses the technology of the low-level visual feature extraction and image similarity measurement, which has poor generalization ability and low retrieval efficiency. In order to obtain more detailed and abundant image features, a method of flower feature extraction based on deep convolution network is proposed. The deep learning model of VGGNet convolution neural network is used to realize flower retrieval. The experimental results of Oxford 102 flower data set show that the method based on VGG16 model has the characteristics of high accuracy, fast query speed and good robustness.
\end{abstract}

Keywords: flower retrieval, deep learning, VGG 16, feature extraction.

\section{Introduction}

Admire the beauty of flowers is a kind of behavior that human beings are close to nature. People's demand for flower appreciation is increasing. When we see a beautiful flower but don't know its name, we can't further understand it and acquire its related knowledge. The most primitive method is that users input text through the network to retrieve, so it depend on whether the text information input by users is accurate. In 1992, Kato first proposed a content-based image retrieval method, which means that the query condition itself is an image. By extracting the color, texture, shape and other features of the image to establish the index, and by comparing the distance between these features to determine the similarity between the instance image and the any image in the image datebase. Ke Xiao et al. ${ }^{(1)}$ researched the region segmentation, feature extraction for flower image and the fusion of the same content-based image filtering and flower image retrieval algorithm based on SVM. K. H. Phyu et al. ${ }^{(2)}$ proposed a feature extraction method based on Canny edge histogram descriptor, color layout descriptor and shape descriptor in curvature scale space, which improved the accuracy of flower retrieval. D. H. Apriyanti et al. ${ }^{(3)}$ had a study on orchid and improved the retrieval rate by using the maximum similarity method based on region merging to segment flower images and extract features. According to the characteristics of flower images Wu Huining ${ }^{(4)}$ come up with an improved visual attention model based on Itti model, which combines the transformed flower saliency map with visual attention model to extract features, and then combines with SVM multi-class classifier to improve the accuracy of flower retrieval. Although some studies have shown that the accuracy of flower classification can be improved to some extent by fusing characteristic information such as color, texture and shape, these methods are difficult to achieve better results because of the complex background, intensity of light, shooting angle, projection and occlusion between flowers and leaves ${ }^{(5)}$.

In 2006, G E Hinton in Toronto University published an article based on "Dimension Decline Based on Neural Networks" ${ }^{(6)}$ which opened the prelude of in-depth learning research. Compared with the existing methods, one direction of current research is to adopt the end-to-end method of deep convolution neural network, which can automatically and efficiently learn and classify image features. In 2012, Alex et al. won the championship in the ImageNet Image Recognition Competition by using CNN, 
and were well ahead of the second place, thus it started the upsurge of CNN research. AlexNet ${ }^{(7), ~ C a f f e N e t, ~}$ GoogleNet ${ }^{(8)}$, VGGNet ${ }^{(9)}$, $\operatorname{ResNet}^{(10)}$ and other network models have made continuous breakthroughs in image classification. The classification accuracy of mainstream models in ImageNet on large-scale image data sets has reached $97 \%$, which exceeds the recognition level of experts. It shows that the convolution neural network method can effectively learn the intrinsic features of images and improve the accuracy of image retrieval. The application of in-depth learning in content-based flower image retrieval has the following advantages ${ }^{(11)}$ : (1) There is no need to preprocess the flower image edge segmentation so that we can avoid the classification error caused by the segmentation. (2) The features extracted automatically by in-depth learning which have strong robustness, and the accuracy of classification is much higher than that of traditional methods. (3) The in-depth learning model has better generalization ability. It can not only extract the underlying semantic features of the image, but also learn the high-level semantic features, which makes the model contain various feature information and have stronger retrieval ability for different data.

This paper uses VGG-Net network model to retrieve, and in ILSVRC location and classification in 2014, the model has won the championship and runner-up respectively. This paper improves the VGG-Net network model. The improved VGG-Net retains the first two full connection layers on the basis of the original model, deletes the last full connection layer, and increases the number of convolution cores in the last module of the model, so as to improve the accuracy of the system. Comparing with CaffeNet, the improved VGG16 has obvious advantages.

\section{Flower Retrieval Based on VGG-Net}

\subsection{Introduction of the original VGG-Net network structure}

\subsubsection{Original VGG-Net Network Architecture}

The standard size RGB image is input into VGG-Net, and the size is $3 \times 3$. The $2 \sim 3$ continuous convolution layer is a unit module in the network then through a series of stacked convolution cores. This unit module is connected with the maximum pooling layer to reduce over-fitting by compressing data and parameters. Through these similar modules, three layers of traditional neural network, i.e. three layers of full connection layer, are connected. Dropout is used in all connection layers, which can effectively alleviate the occurrence of over-fitting, achieve regularization effect to a certain extent, and the generalization ability of the model is also strong. Finally, it is connected with the Softmax classifier. VGG-Net uses smaller convolution and pooling cores. The deeper layers can make the model converge faster and have better learning ability, so it has strong extensibility and good generalization. The network structure is shown in Figure 1 below.

\subsubsection{ReLU Activation function}

VGG-Net uses ReLU activation function to calculate nonlinearity. Its activation function model is similar to that of human brain neurons. This functional model has three main characteristics: unilateral inhibition, relatively wide excitation boundary and sparse activation.

The function expression is shown in Eq.1.

$$
f(x)=\max (0, x)
$$

When the input $x$ value is less than 0 , the output value is 0 . When the input $x$ value is more than 0 , the output value is equal to the input value, which reflects the choice of the ReLU activation function to the signal. This feature can shield part of the noise mixed in the signal, not only can improve the learning accuracy, but also can obtain sparse signal characteristics.

\subsubsection{Dropout}

Overfitting is a common problem in deep learning. If the model is overfitted, the adopted model is almost useless. In order to solve the problem of overfitting, multiple model integration methods can be adopted. But training multiple models and testing multiple models can be cumbersome. Overfitting and time consuming are two major shortcomings of training depth learning, and dropout can effectively alleviate overfitting and achieve regularization effect to a certain extent. 


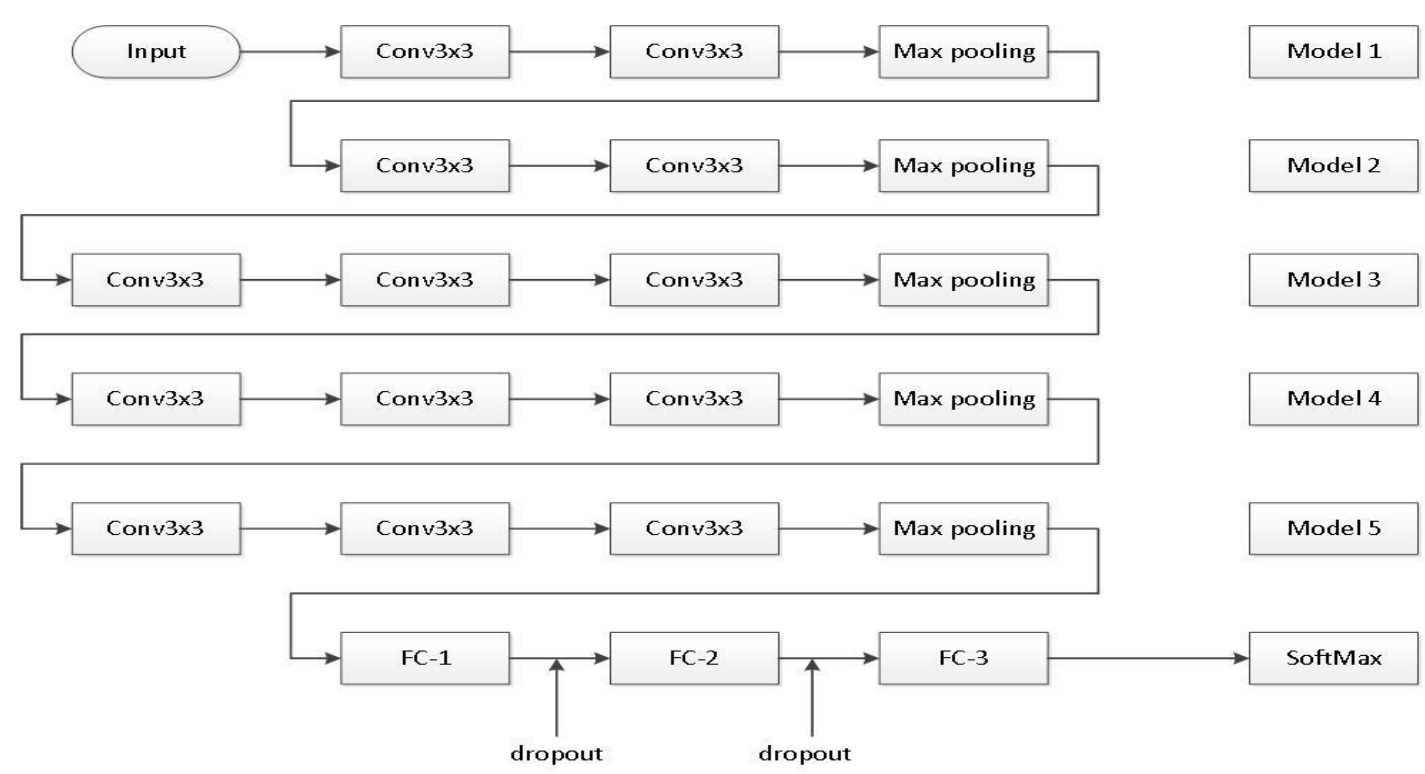

Fig. 1. VGG network architecture

In 2012, dropout was proposed by Hinton in his thesis. During the forward propagation, the dropout could make the activation value of a neuron stop working with a certain probability $P$, so that it would not rely too much on some local characteristics and improve the generalization of the model. The model is shown in Fig. 2.

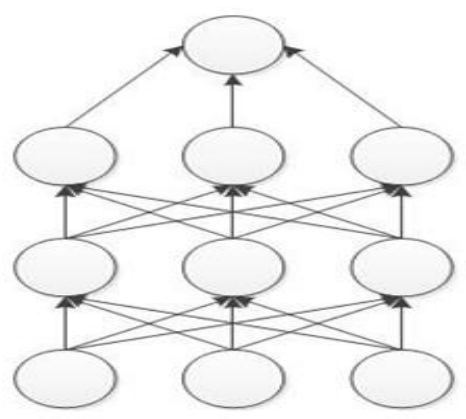

(a) all the connection

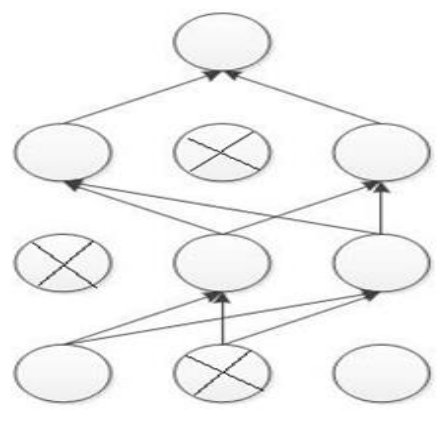

(b) part of the connection

Fig.2. Dropout model diagram

In Fig.2, the neuron layers in subfigure (a) are connected with each other, and the neuron layers in subfigure (b) choose to connect with each other, and some neurons stop working. This reduces the number of weights and bias parameters in some neurons and prevents over-fitting of input sample.

\section{Improved VGG-net network model structure}

The VGG16 neural network contains about 138 million parameters. Corresponding to each neuron in the sample training process, we should save the weights and the partial derivatives of model's bias. In order to obtain more detailed flower image features, this paper makes following three improvements on the original VGG-16 model. The specific network parameters are shown in Table 1.

Firstly, the convolution kernel number in conv4 and conv5 layers was changed from 512 to 256, which reduced the number of parameters and the training time of the network without affecting the accuracy, and prevented the overfitting of the model to some extent.

Secondly, on the basis of VGG16 structure, 1000 output nodes of full-connection layers were changed to 102 nodes. So the number of final categories, parameters and training time are reduced and extract accuracy is improved.

Thirdly, the average pooling layer will be used to replace the Max pooling layer in conv5layer. This method extracts more detailed image features without affecting network performance, and slightly improves learning time to obtain more abstract image representation without changing the computation amount of data to be saved in module 5 . 
Table 1. Parameters of VGG network after improvement

\begin{tabular}{|c|c|c|c|c|c|}
\hline & Conv3x3 & Conv $3 \times 3$ & Conv $3 \times 3$ & pooling & output size \\
\hline Input & \multicolumn{5}{|c|}{$3 \times 244 \times 244$} \\
\hline Module 1 & $\begin{array}{l}\text { filter size: } 3 \times 3 \\
\text { filter number: } 64\end{array}$ & $\begin{array}{l}\text { filter size: } 3 \times 3 \text { filter } \\
\text { number: } 64\end{array}$ & & $\begin{array}{c}\text { type: Max } \\
\text { filter size: } 2 \times 2\end{array}$ & $112 \times 112 \times 64$ \\
\hline Module 2 & $\begin{array}{c}\text { filter size: } 3 \times 3 \\
\text { filter number: } 128\end{array}$ & $\begin{array}{c}\text { filter size: } 3 \times 3 \\
\text { filter number: } 128\end{array}$ & & $\begin{array}{c}\text { type: Max } \\
\text { filter size: } 2 \times 2\end{array}$ & $56 \times 56 \times 128$ \\
\hline Module 3 & $\begin{array}{c}\text { filter size: } 3 \times 3 \\
\text { filter number: } 256\end{array}$ & $\begin{array}{c}\text { filter size: } 3 \times 3 \\
\text { filter number: } 256\end{array}$ & $\begin{array}{c}\text { filter size: } 3 \times 3 \\
\text { filter number: } 256\end{array}$ & $\begin{array}{c}\text { type: } \text { Max } \\
\text { filter size: } 2 \times 2\end{array}$ & $28 \times 28 \times 256$ \\
\hline Module 4 & $\begin{array}{c}\text { filter size: } 3 \times 3 \\
\text { filter number: } 256\end{array}$ & $\begin{array}{c}\text { filter size: } 3 \times 3 \\
\text { filter number: } 256\end{array}$ & $\begin{array}{c}\text { filter size: } 3 \times 3 \\
\text { filter number: } 256\end{array}$ & $\begin{array}{c}\text { type: Max } \\
\text { filter size: } 2 \times 2\end{array}$ & $14 \times 14 \times 256$ \\
\hline Module 5 & $\begin{array}{c}\text { filter size: } 3 \times 3 \\
\text { filter number: } 256\end{array}$ & $\begin{array}{c}\text { filter size: } 3 \times 3 \\
\text { filter number: } 256\end{array}$ & $\begin{array}{c}\text { filter size: } 3 \times 3 \\
\text { filter number: } 256\end{array}$ & $\begin{array}{l}\text { type: Average } \\
\text { filter size: } 2 \times 2\end{array}$ & $7 \times 7 \times 256$ \\
\hline FC 1 & \multicolumn{4}{|c|}{4096 Output node } & $1 \times 1 \times 4096$ \\
\hline FC 2 & \multicolumn{4}{|c|}{4096 Output node } & $1 \times 1 \times 4096$ \\
\hline FC 3 & \multicolumn{4}{|c|}{1000 Output node } & $1 \times 1 \times 102$ \\
\hline \multicolumn{6}{|c|}{ Softmax } \\
\hline
\end{tabular}

\section{Experimental results and analysis}

\subsection{Flower Database}

Currently, there are relatively few flower data sets which are publicly available on the Internet. In this experiment, Oxford 102 Flowers data set was used. The data set contains 102 categories of flowers and a total of 8,189 images. Most of the flowers are grown in the UK. Most of the them are from the Internet and a small number of them have been filmed by researchers. Each type of flower with different sizes has at least 40 images, and at most 250 images. The data set consists of 1020 training sets, 1020 verification sets and 6149 test sets. Compared with other flower data sets, Oxford 102 Flowers has a better experimental effect.

\subsection{Experimental Environment Setting}

In order to improve the accuracy of flower retrieval by improving VGG16 structure. In this experiment, under the ubuntu16.04 system, the graphics card uses Nvidia-1080ti and takes TensorFlow as the back end. In Keras, the Oxford102 flower model was trained based on the VGG16 model. Finally, specific parameters were set: batch size was 32 , learning rate was $10^{-5}$, weight decay was $5 \times 10^{-4}$, and inertia momentum was 0.9 . In terms of input data, a random clipping method is adopted to obtain fixed size $224 \times 224$ as the input image, and a random horizontal mirror image and a random translation image channel are adopted to enrich the data. In terms of evaluation indexes, the retrieval accuracy and the average training time of each image were measured.

\subsection{Experimental Results and Analysis}

Experiment 1: Visualization of flower feature map based on deconvolution network.

With the visualization of convolutional neural network, the features learned at each layer of $\mathrm{CNN}$ can be clearly seen, and then the network and parameters can be adjusted through visualization to improve the accuracy of the model. Visualization is performed by deconvolution of feature maps output on each convolution layer. The deconvolution network can be seen as the inverse process of the convolution network, which is caused depooling, deactivation and deconvolution.

If you want to see what was extracted from conv5 layer in VGG16 model, connect a deconvolution network through the conv5 feature map. You can enlarge the original size $(7 \times 7)$ of the feature map to get an image which is the same size as the original image $(224 \times 224)$.

Fig. 3 shows 5 images selected from the training set, and Fig. 4 shows visualized images output by each layer of VGG network. 


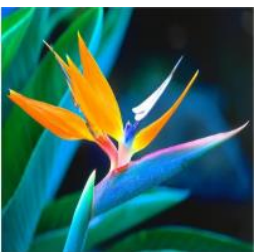

(a) Flowers 1

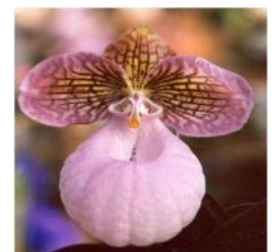

(b) Flowers 2

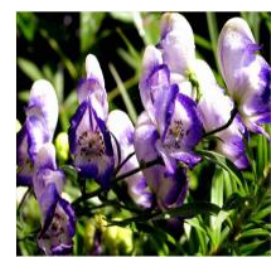

(c) Flowers 3

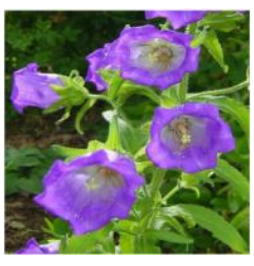

(d) Flower 4

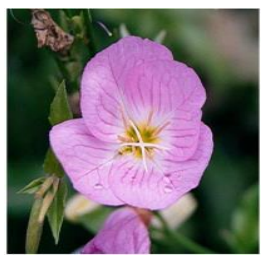

(e) Flowers 5
Fig.3. Select 5 flower images

As can be seen from Fig. 4, deeper output extracts more advanced semantic features to better locate and highlight the target. The lateral output of the shallower layer can locate abundant spatial information. After learning from $\mathrm{CNN}$, the features are discriminant, and the activation degree of background is basically very low. The extracted features are visualized and the background is ignored, but the key information is extracted. The features learned from layer 1 and layer 2 are basically low-level features such as color and edge. Layer 3 starts to get a little bit more complicated features which are learned from texture features like some of the meshes. Layer 4 and layer 5 learn details that are more semantic and have critical distinguishing features.

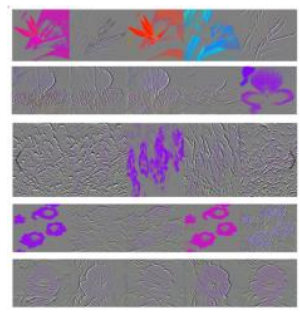

(a) Layer 1

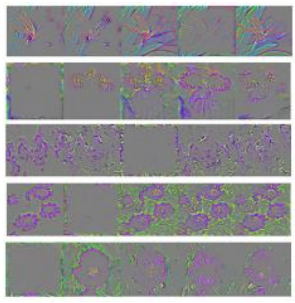

(c) Layer 3

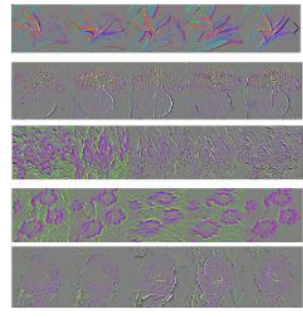

(b) Layer 2

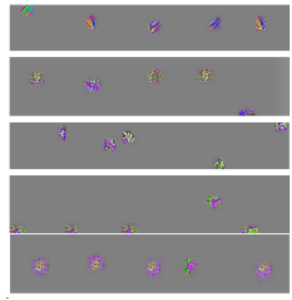

(d) Layer 4

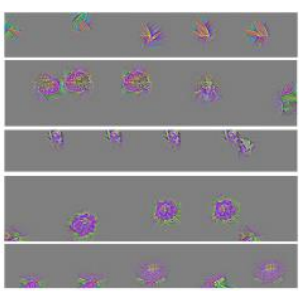

(e) Layer 5

Fig.4. Visual images output by each layer of VGG network

Experiment 2: Retrieving by an instance image.

The retrieval system is set to retrieve 10 similar images for every input instance image. Fig.5 and Fig.7 are input instance images, and Fig. 6 and Fig. 8 are other retrieved images.

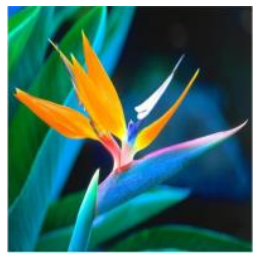

Fig.5. Instance image 1

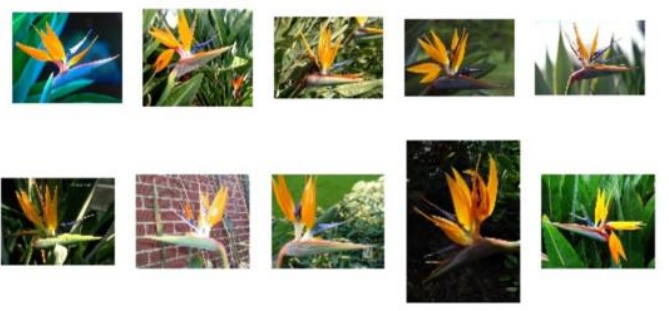

Fig.6. Search result 1

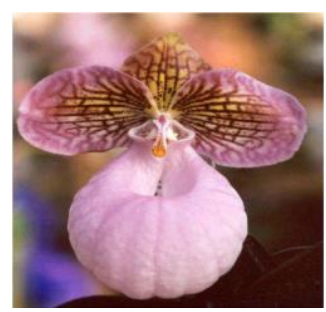

Fig.7. Instance image 2

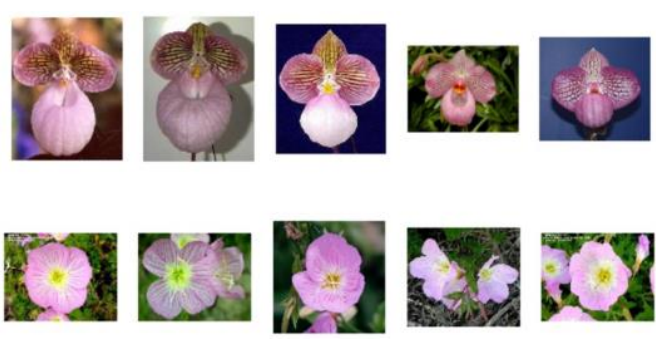

Fig.8. Search result 2 
As shown in Table 2, the retrieval result accuracy increased from $81.43 \%$ to $86.47 \%$ by $5.03 \%$ with the method in this paper. The processing time for each image in the data set was $0.732 \mathrm{~s}$. Fig. 9 records the real-time relationship between the number of iterations and test accuracy.

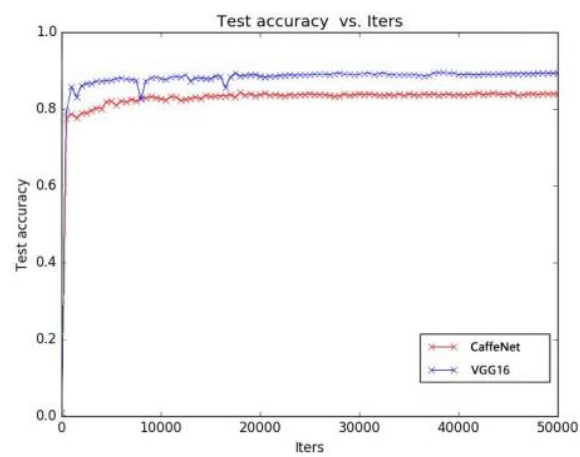

Fig.9. CaffeNet and VGG16 models' accuracy is related to the number of iterations

Table 2. CaffeNet and VGG16 model accuracy and processing time per image

\begin{tabular}{|c|r|c|}
\hline Model & $\begin{array}{r}\text { Accuracy } \\
\text { of the test }\end{array}$ & $\begin{array}{c}\text { Average processing } \\
\text { time per image }\end{array}$ \\
\hline CaffeNet & $81.43 \%$ & $0.846 \mathrm{~s}$ \\
\hline VGG16 & $86.47 \%$ & $0.732 \mathrm{~s}$ \\
\hline
\end{tabular}

\section{Conclusions}

This paper compares CaffeNet's algorithm with the proposed algorithm which adjusts the VGG-net network model and parameters, and verifies the feasibility of this algorithm. After many experiments with the data set of Oxford 102 Flowers, the results can prove that the precision of flower retrieval has been improved obviously.

\section{Acknowledgment}

The writers would like to extend their thanks to the anonymous reviewers for their extremely useful advice and comments to improve this paper. This work was supported by Jiangsu Province 7th Projects for "Summit Talents in Six Main Industries"; Electronic Information Industry, DZXX-149, No.110); the National Natural Science Foundation of China (Grant No. 61802336); 2014 Jiangsu
Province Enterprise Postgraduate Student Workstation at Jiangsu Haorun Electronic Technology Co., Ltd.

\section{References}

(1) Ke Xiao, Chen Xiaofen, and Li Shaozi : "Flower Image Retrieval Based on Multi-features Fusion", Journal of Computer Science, Vol. 37, No. 11, pp. 282-286, 2010

(2) K. H. Phyu and A. Kutics and A. Nakagawa : "Self-adaptive Feature Extraction Scheme for Mobile Image Retrieval of Flowers", SITIS Transactions, pp. 366-373, 2012

(3) D. H. Apriyanti and A. M. Arymurthy and L. T. Handoko : "Identification of orchid species using content-based flower image retrieval", IC3INA Transactions, pp. 53-57, 2013

(4) Wu Huining, $\mathrm{Hu}$ Xuelong, and Lu Huimin: "Image retrieval based on information entropy and block color moments", Journal of Yangzhou University, Vol. 17, No.3, pp. 50-53, 2010

(5) Zhu Danhong, Ke Xiao, and Ye Jing : "Particle swarm optimization combined with relevance feedback for flower image retrieval", Journal of Fuzhou University, Vol. 44, No. 2, pp. 182-187, 2016

(6) Hinton G E, and Salakhutdinov R R : "Reducing the dimensionality of data with neural networks.”, Journal of Science, Vol. 313, No. 5786, pp. 504-507, 2006

(7) Krizhevsky A, Sutskever I, and Hinton G E : "ImageNet classification with deep convolution neural networks", NIPS Transactions, Vol. 25, 2012

(8) C. Szegedy and Wei Liu and Yangqing Jia and P. Sermanet and S. Reed and D. Anguelov and D. Erhan and V. Vanhoucke and A. Rabinovich : "Going deeper with convolutions", CVPR Transactions, pp. 1-9, 2015

(9) Simonyan K, and Zisserman, A : "Very Deep Convolutional Networks for Large-Scale Image Recognition", Journal of Computer Science, 2014

(10)He K, Zhang X, Ren S, and Sun J : “Deep Residual Learning for Image Recognition”, CVPR Transactions, pp. 770-778, 2016

(11) $\mathrm{Hu}$ Erlei, and Feng Rui : "Image Retrieval System Based on Deep Learning”, Journal of Application of Computer System, Vol. 26, No. 3, pp. 8-19, 2017 